\title{
Some generalized three-term conjugate gradient methods based on CD approach for unconstrained optimization problems
}

\author{
Ladan Arman*, Yuanming Xu*, Long Liping**1 \\ *School of Aeronautic Science and Engineering, Beihang University, Beijing, China \\ **Institute of Mechanics, Chinese Academy of Sciences, Beijing 100190, China \\ lplong05@163.com
}

Abstract. In this paper, based on the efficient Conjugate Descent (CD) method, two generalized CD algorithms are proposed to solve the unconstrained optimization problems. These methods are threeterm conjugate gradient methods which the generated directions by using the conjugate gradient parameters and independent of the line search satisfy in the sufficient descent condition. Furthermore, under the strong Wolfe line search, the global convergence of the proposed methods is proved. Also, the preliminary numerical results on the CUTEst collection are presented to show effectiveness of our methods.

Keywords. Conjugate gradient method, Unconstrained optimization, Global convergence, Strong Wolfe line search.

\section{Introduction}

Consider the following unconstrained optimization problem

$$
\min f(x), \quad x \in \mathbb{R}^{n}
$$

where $f: \mathbb{R}^{n} \rightarrow \mathbb{R}$ is a continuously differentiable function and its gradient $g:=\nabla f$ is available. Conjugate Gradient (CG) methods are effective iterative methods for solving (1), especially for largescale problems. The important properties of these methods are the use only first-order derivatives, little storage and computation requirements, and strong local and global convergence properties $[1,9,18,22]$. Starting from an initial guess $x_{0} \in \mathbb{R}^{n}$, the CG methods generate a sequence $\left\{x_{k}\right\}_{k \geq 0}$ as

$$
x_{k+1}=x_{k}+\alpha_{k} d_{k}
$$

where $\alpha_{k}>0$ is step-length and usually obtained using some inexact line search. Furthermore, $d_{k}$ is the search direction calculated by

$$
d_{k}= \begin{cases}-g_{k}, & k=0 \\ -g_{k}+\beta_{k} d_{k-1}, & k>0\end{cases}
$$

in which $g_{k}=g\left(x_{k}\right)$ and $\beta_{k}$ is a scalar. There are many variants of CG methods, which are obtained with different choices for the parameter $\beta_{k}$. The most important CG methods proposed by FletcherReeves (FR) [16], Hestenes-Stiefel (HS) [19], Conjugate Descent (CD) by Fletcher [15], Polak-RibierePolyak (PRP) [22, 23], Dai-Yuan (DY) [10] and Hager-Zhang (HZ) [17] are defined by

\footnotetext{
${ }^{1}$ Corresponding author
} 


$$
\begin{aligned}
& \beta_{k}^{F R}=\frac{\left\|g_{k}\right\|^{2}}{\left\|g_{k-1}\right\|^{2}}, \quad \beta_{k}^{H S}=\frac{g_{k}^{T} y_{k-1}}{d_{k-1}^{T} y_{k-1}} \quad \beta_{k}^{C D}=-\frac{\left\|g_{k}\right\|^{2}}{g_{k-1}^{T} d_{k-1}} \\
& \beta_{k}^{P R P}=\frac{g_{k}^{T} y_{k-1}}{\left\|g_{k-1}\right\|^{2}}, \quad \beta_{k}^{D Y}=\frac{\left\|g_{k}\right\|^{2}}{d_{k-1}^{T} y_{k-1}}, \quad \beta_{k}^{H Z}=\left(y_{k-1}-2 d_{k-1} \frac{\left\|y_{k-1}\right\|^{2}}{d_{k-1}^{T} y_{k-1}}\right)^{T} \frac{g_{k}}{d_{k-1}^{T} y_{k-1}},
\end{aligned}
$$

in which $\|\cdot\|$ is the Euclidean norm and $y_{k-1}=g_{k}-g_{k-1}$. These methods are the identical where the objective function $f$ is quadratic and exact line search is used [21], but for general objective function the behaviour of these methods is different.

Generally, in the iterative methods, we need the search direction $d_{k}$ satisfy the descent condition

$$
g_{k}^{T} d_{k}<0, \quad \forall k \geq 0 .
$$

In order to guarantee the lobal convergence of CG methods, the direction $d_{k}$ must satisfy the sufficient descent condition

$$
g_{k}^{T} d_{k}<-c\left\|g_{k}\right\|^{2}, \quad \forall k \geq 0,
$$

in which $c$ is a positive constant. There are many CG methods which satisfy (7), see $[3,17,20]$.

In practical the step-size $\alpha_{k}$ is determined by inexact line search. Some inexact line search techniques have been provided in [21]. The standard Wolfe conditions are [24]

$$
\begin{aligned}
& f\left(x_{k}+\alpha_{k} d_{k}\right)-f\left(x_{k}\right) \leq c_{1} \alpha_{k} g_{k}^{T} d_{k}, \\
& g_{k+1}^{T} d_{k} \geq c_{2} g_{k}^{T} d_{k},
\end{aligned}
$$

where $\cdot<c_{1}<c_{2}<1$. To convergence analysis and numerical implementations of CG methods, the step-size $\alpha_{k}$ is often obtained from the strong Wole line search [25] by

$$
\begin{aligned}
& f\left(x_{k}+\alpha_{k} d_{k}\right)-f\left(x_{k}\right) \leq c_{1} \alpha_{k} g_{k}^{T} d_{k}, \\
& \left|g_{k+1}^{T} d\right|_{k} \leq-c_{2} g_{k}^{T} d_{k} .
\end{aligned}
$$

Furthermore, the generalized Wolfe conditions for $\cdot<c_{1}<c_{3}<1$ and $c_{4} \geq 0$ are as follows:

$$
\begin{aligned}
& f\left(x_{k}+\alpha_{k} d_{k}\right)-f\left(x_{k}\right) \leq c_{1} \alpha_{k} g_{k}^{T} d_{k}, \\
& c_{3} g_{k}^{T} d_{k} \leq g_{k+1}^{T} d_{k} \leq-c_{4} g_{k}^{T} d_{k} .
\end{aligned}
$$

For the first time, the general three-term conjugate gradient (TTCG) methods were proposed by Beale [7] to solve the unconstrained optimization problems. In this approach, the search direction $d_{k}$ is

$$
d_{k}=-g_{k}+\beta_{k} d_{k-1}+\gamma_{k} d_{t},
$$

where $\beta_{k}=\beta_{k}^{F R}, \beta_{k}^{H S}, \beta_{k}^{D Y}$. Furthermore, $d_{t}$ is a restart direction and

$$
\gamma_{k}= \begin{cases}0, & k=t+1, \\ \frac{g_{k}^{T} y_{t}}{d_{t}^{T} y_{t}}, & k>t+1 .\end{cases}
$$

However, TTCG methods are obtained to improve traditional conjugate gradient methods and different choices for three-term conjugate gradient parameters lead to different TTCG methods. Further efforts 
have been made to develop the TTCG methods with the sufficient descent property $[2,6,26]$, the descent and conjugacy properties [4,11] and the sufficient descent and conjugacy properties [13,14]. A comparison between some TTCG methods is reported for solving unconstrained optimization problems, see [5].

In this paper, we introduce two three-term conjugate gradient methods based on CD algorithm. Also, the generated search directions satisfy the sufficient descent property, independent of line search. The global convergence of the new methods is proven for general functions under mild assumptions. Also, numerical experiments confirm that our methods are efficient to solve unconstrained optimization problems in compared to some conjugate gradient method.

The structure of this paper is as follows. In Section 2, we propose two generalize of CD algorithm which are TTCG methods. The sufficient descent property of generated directions and the global convergence of the proposed algorithms are established in Section 3. In Section 4, we provide some numerical experiments to demonstrate the efficiency of our methods. Finally, some conclusions are given in Section 5.

\section{Motivation and the new algorithms}

In this section, we introduce two three-term conjugate gradient algorithms to solve unconstrained optimization problem (1) based on CD method. Fletcher in [15] proposed the CD conjugate gradient method which is closely related to the FR method. Note that to obtain the step-length $\alpha_{k}$, we should solve the following one-dimensional optimization problem

$$
\alpha_{k}=\underset{\alpha>0}{\arg \min } f\left(x_{k}+\alpha d_{k}\right) \text {. }
$$

The $\mathrm{CD}$ conjugate gradient method is equal to FR conjugate gradient method when the exact line search is used. The exact line search implies $g_{k+1}^{T} d_{k}=0$. Therefore, from (3), we get

$$
g_{k-1}^{T} d_{k-1}=g_{k-1}^{T}\left(-g_{k-1}+\beta_{k-1} d_{k-2}\right)=-\left\|g_{k-1}\right\|^{2}+\beta_{k-1} g_{k-1}^{T} d_{k-2}=-\left\|g_{k-1}\right\|^{2} .
$$

Hence

$$
\beta_{k}^{F R}=\frac{\left\|g_{k}\right\|^{2}}{\left\|g_{k-1}\right\|^{2}}=-\frac{\left\|g_{k}\right\|^{2}}{g_{k-1}^{T} d_{k-1}}=\beta_{k}^{C D} .
$$

On the other hand, the generated directions by CD method satisfy the sufficient descent condition with strong Wolfe line search [18]. Also, from the generalized Wolfe condition with $c_{3}<1$ and $c_{4}=0$, we obtain $0 \leq \beta_{k}^{C D} \leq \beta_{k}^{F R}$. Hence, the global convergence of CD method will be obtained by Theorem 2.2 in [1]. Now, we generalize the CD method to obtain a new three-term conjugate gradient method (NTTCD) where the direction $\mathrm{dk}$ is calculated by

$$
d_{k}=\left\{\begin{array}{lr}
-g_{k}, & k=0, \\
-g_{k}+\beta_{k}^{C D} d_{k-1}+\theta_{k} g_{k}, & k \geq 1,
\end{array}\right.
$$

where the parameter $\theta_{k}$ is to grantee the sufficient descent condition and defined by

$$
\theta_{k}=\frac{g_{k}^{T} d_{k-1}}{g_{k-1}^{T} d_{k-1}}
$$

We will show that the search direction (16) satisfies $g_{k}^{T} d_{k}=-\left\|g_{k}\right\|^{2}$, independent of the line search and the objective function convexity. Furthermore, using the exact line search NTTCD method is reduced to CD method. To augment the efficiency of NTTCD method, we consider the following 
modification of this method. Hence, we get MNTTCD method while the search direction is generated by

$$
d_{k}=\left\{\begin{array}{lr}
-g_{k}, & k=0, \\
-g_{k}+\beta_{k}^{C D} d_{k-1}+t_{k} \theta_{k} g_{k}, & k \geq 1,
\end{array}\right.
$$

in which

$$
t_{k}= \begin{cases}\max \left\{1, \min \left\{\eta_{1}, \frac{g_{k}^{T} d_{k-1}}{\max \left\{\zeta_{1},\left\|y_{k-1}\right\|\left\|d_{k-1}\right\|\right\}}\right\}\right\}, & g_{k}^{T} d_{k-1}>0, \\ \max \left\{\eta_{2}, \frac{g_{k}^{T} d_{k-1}}{\max \left\{\zeta_{2},\left\|y_{k-1}\right\|\left\|d_{k-1}\right\|\right\}}\right\}, & g_{k}^{T} d_{k-1} \leq 0,\end{cases}
$$

where $\eta_{2}<0<\eta_{1}$ and $\zeta_{1}, \zeta_{2}>0$ are constant. Note that for $t_{k}=0$ and $t_{k}=1$ the MNTTCD method reduces to CD and NTTCD methods, respectively.

Now, we present the structure of new three-term conjugate gradient algorithms as follows:

\section{Algorithm 1: The new three-term conjugate gradient method (NTTCD)}

Step 0: Choose positive constant $\varepsilon, 0<c_{1}<c_{2}<1$ and an initial point $x_{0} \in \mathbb{R}^{n}$. Set $k=0$, $d_{0}=-g_{0}$.

Step 1: Terminate the algorithm once $\left\|g_{k}\right\| \leq \varepsilon$ holds.

Step 2: Find the step-length $\alpha_{k}$ satisfying the strong Wolfe condition (10)-(11).

Step 3: Generate the new iterate by $x_{k+1}=x_{k}+\alpha_{k} d_{k}$.

Step 4: Calculate $g_{k+1}$ and the conjugate parameter $\beta_{k+1}^{C D}$ by (4).

Step 5: Obtain the parameter $\theta_{k+1}$ with (17) and the new search direction $d_{k+1}$ by (16).

Step 6: Set $k=k+1$ and go to Step 1 .

\section{Algorithm 2: The modification of new three-term conjugate gradient method (MNTTCD)}

Step 0: Choose positive constant $\varepsilon, \zeta_{1}, \zeta_{2}, \eta_{2}<0<\eta_{1}, 0<c_{1}<c_{2}<1$ and an initial point $x_{0} \in \mathbb{R}^{n}$. Set $k=0, d_{0}=-g_{0}$.

Step 1: Terminate the algorithm once $\left\|g_{k}\right\| \leq \varepsilon$ holds.

Step 2: Find the step-length $\alpha_{k}$ satisfying the strong Wolfe condition (10)-(11).

Step 3: Generate the new iterate by $x_{k+1}=x_{k}+\alpha_{k} d_{k}$.

Step 4: Calculate $g_{k+1}$ and the conjugate parameter $\beta_{k+1}^{C D}$ by (4).

Step 5: Obtain the parameter $\theta_{k+1}$ with (17), $t_{k}$ by (19) and the new search direction $d_{k+1}$ by (18). 
Step 6: Set $k=k+1$ and go to Step 1 .

\section{Convergence analysis}

In this section, the sufficient descent property and the global convergence of the new algorithms are established. To this aim, we make some assumptions on the objective function as follows:

Assumption 3.1 The level set $L\left(x_{0}\right)=\left\{x \in \mathbb{R}^{n} \mid f(x) \leq f\left(x_{0}\right)\right\}$ is bounded, i.e., there exists a constant $M>0$ such that

$$
\|x\| \leq M, \quad \forall x \in L\left(x_{0}\right) .
$$

Assumption 3.2 In some neighborhood $\Omega \subseteq L\left(x_{0}\right)$ the gradient of the objective function $f$ is Lipschitz continuous, i.e., there exists a constant $L>0$ such that

$$
\|g(x)-g(y)\| \leq L\|x-y\|, \quad \forall x, y \in \Omega .
$$

Lemma 1 Suppose that $\left\{d_{k}\right\}_{k \geq 0}$ is generated by NTTCD algorithm. Then, we have

$$
g_{k}^{T} d_{k}=-\left\|g_{k}\right\|^{2} \text {. }
$$

Proof: By multiplying (16) in $g_{k}^{T}$, using (4) and (17), we obtain

$$
\begin{aligned}
g_{k}^{T} d_{k} & =-\left\|g_{k}\right\|^{2}+\beta_{k}^{C D} g_{k}^{T} d_{k-1}+\theta_{k}\left\|g_{k}\right\|^{2} \\
& =-\left\|g_{k}\right\|^{2}-\frac{\left\|g_{k}\right\|^{2}}{g_{k-1}^{T} d_{k-1}} g_{k}^{T} d_{k-1}+\frac{g_{k}^{T} d_{k-1}}{g_{k-1}^{T} d_{k-1}}\left\|g_{k}\right\|^{2} \\
& =-\left\|g_{k}\right\|^{2}<0 .
\end{aligned}
$$

Therefore, the proof is complete.

Lemma 2 Let $\left\{d_{k}\right\}_{k \geq 0}$ be generated direction by MNTTCD algorithm. Then, $\left\{d_{k}\right\}_{k \geq 0}$ satisfy the sufficient descent condition (7) with $c=1$, i.e.

$$
g_{k}^{T} d_{k} \leq-\left\|g_{k}\right\|^{2} \text {. }
$$

Proof: We prove this lemma in two following cases.

Case (1): Let $g_{k}^{T} d_{k-1}>0$. From (4), (17) and (18), we get

$$
g_{k}^{T} d_{k}=-\left\|g_{k}\right\|^{2}-\frac{\left\|g_{k}\right\|^{2}}{g_{k-1}^{T} d_{k-1}} g_{k}^{T} d_{k-1}+t_{k} \frac{g_{k}^{T} d_{k-1}}{g_{k-1}^{T} d_{k-1}}\left\|g_{k}\right\|^{2} .
$$

Using (19), there are two choices for parameter $t_{k}$.

(i) For $t_{k}=1$, we have

$$
g_{k}^{T} d_{k}=-\left\|g_{k}\right\|^{2}-\frac{\left\|g_{k}\right\|^{2}}{g_{k-1}^{T} d_{k-1}} g_{k}^{T} d_{k-1}+\frac{g_{k}^{T} d_{k-1}}{g_{k-1}^{T} d_{k-1}}\left\|g_{k}\right\|^{2}=-\left\|g_{k}\right\|^{2}<0 .
$$


(ii) If $\min \left\{\eta_{1}, \frac{g_{k}^{T} d_{k-1}}{\max \left\{\zeta_{1},\left\|y_{k-1}\right\|\left\|d_{k-1}\right\|\right\}}\right\}>1$, then we use induction over $k$ to prove this item.

Now, induction hypothesis implies $g_{k-1}^{T} d_{k-1} \leq-\left\|g_{k-1}\right\|^{2}<0$. Therefore, we have

Hence

$$
\frac{g_{k}^{T} d_{k-1}}{g_{k-1}^{T} d_{k-1}}\left\|g_{k}\right\|^{2}<0
$$

$$
t_{k} \frac{g_{k}^{T} d_{k-1}}{g_{k-1}^{T} d_{k-1}}\left\|g_{k}\right\|^{2}<\frac{g_{k}^{T} d_{k-1}}{g_{k-1}^{T} d_{k-1}}\left\|g_{k}\right\|^{2} .
$$

So, (24) and (25) give us

$$
g_{k}^{T} d_{k} \leq-\left\|g_{k}\right\|^{2}-\frac{\left\|g_{k}\right\|^{2}}{g_{k-1}^{T} d_{k-1}} g_{k}^{T} d_{k-1}+\frac{g_{k}^{T} d_{k-1}}{g_{k-1}^{T} d_{k-1}}\left\|g_{k}\right\|^{2}=-\left\|g_{k}\right\|^{2}<0 .
$$

Therefore, for this case $d_{k}$ satisfy the sufficient descent condition.

Case (2): If $g_{k}^{T} d_{k-1} \leq 0$, then

$$
t_{k}=\max \left\{\eta_{2}, \frac{g_{k}^{T} d_{k-1}}{\max \left\{\zeta_{2},\left\|y_{k-1}\right\|\left\|d_{k-1}\right\|\right\}}\right\} \leq 0
$$

Similar to case (1), using induction over $k$, we have $g_{k-1}^{T} d_{k-1} \leq-\left\|g_{k-1}\right\|^{2}<0$. Hence

yielding

$$
\frac{g_{k}^{T} d_{k-1}}{g_{k-1}^{T} d_{k-1}}\left\|g_{k}\right\|^{2} \geq 0,
$$

$$
t_{k} \frac{g_{k}^{T} d_{k-1}}{g_{k-1}^{T} d_{k-1}}\left\|g_{k}\right\|^{2} \leq 0 .
$$

Finally, from (24), (26) and (27), we obtain

So, we obtain desired result.

$$
g_{k}^{T} d_{k} \leq-\left\|g_{k}\right\|^{2}<0
$$

Lemma 3 Let $\left\{d_{k}\right\}_{k \geq 0}$ be a sufficient descent direction and the step-length $\alpha_{k}$ satisfies the strong Wolfe line search (10)-(11). Then, based on Assumptions 3.1 and 3.2, we have

$$
\sum_{k=0}^{\infty} \frac{\left(g_{k}^{T} d_{k}\right)^{2}}{\left\|d_{k}\right\|^{2}}<+\infty .
$$


Proof: See [27].

Lemma 4 Under strong Wolfe line search (10)-(11), the parameter $\theta_{k}$ satisfies

$$
-1 \leq \theta_{k} \leq 1 \text {. }
$$

Proof: From (11), it is clear that

$$
c_{2} g_{k-1}^{T} d_{k-1} \leq g_{k}^{T} d_{k-1} \leq-c_{2} g_{k-1}^{T} d_{k-1} .
$$

Since $g_{k-1}^{T} d_{k-1} \leq-\left\|g_{k-1}\right\|^{2}<0$, we get

and

$$
\theta_{k}=\frac{g_{k}^{T} d_{k-1}}{g_{k-1}^{T} d_{k-1}} \leq \frac{c_{2} g_{k-1}^{T} d_{k-1}}{g_{k-1}^{T} d_{k-1}}=c_{2}<1,
$$

Hence

$$
\theta_{k}=\frac{g_{k}^{T} d_{k-1}}{g_{k-1}^{T} d_{k-1}} \geq-\frac{c_{2} g_{k-1}^{T} d_{k-1}}{g_{k-1}^{T} d_{k-1}}=-c_{2}>-1 .
$$

$$
-1 \leq \theta_{k} \leq 1
$$

Theorem 1 Let $\left\{d_{k}\right\}_{k \geq 0}$ be a sufficient descent direction and $\left\{x_{k}\right\}_{k \geq 0}$ be the generated sequence by NTTCD algorithm. Moreover, suppose that the Assumptions 3.1 and 3.2 hold. Then

$$
\lim _{k \rightarrow \infty} \inf \left\|g_{k}\right\|=0 \text {. }
$$

Proof: By contradiction there exists $\varepsilon_{1}>0$ such that $\left\|g_{k}\right\|>\varepsilon_{1}$ for any $k$. So

$$
\frac{1}{\left\|g_{k}\right\|^{2}}>\frac{1}{\varepsilon_{1}^{2}} \text {. }
$$

From (16), we get

Now, (4), (17) and (22) imply

$$
d_{k}=\left(\theta_{k}-1\right) g_{k}+\beta_{k}^{C D} d_{k-1} \text {. }
$$

$$
\begin{aligned}
\left\|d_{k}\right\|^{2} & =\left(\theta_{k}-1\right)^{2}\left\|g_{k}\right\|^{2}+\left(\beta_{k}^{C D}\right)^{2}\left\|d_{k-1}\right\|^{2}+2\left(\theta_{k}-1\right) \beta_{k}^{C D} g_{k}^{T} d_{k-1} \\
& =\left(\theta_{k}-1\right)^{2}\left\|g_{k}\right\|^{2}+\frac{\left\|g_{k}\right\|^{4}}{\left(g_{k-1}^{T} d_{k-1}\right)^{2}}\left\|d_{k-1}\right\|^{2}-2\left(\theta_{k}-1\right) \frac{\left\|g_{k}\right\|^{2}}{g_{k-1}^{T} d_{k-1}} g_{k}^{T} d_{k-1} \\
& =\left(\theta_{k}-1\right)^{2}\left\|g_{k}\right\|^{2}+\frac{\left\|g_{k}\right\|^{4}}{\left\|g_{k-1}\right\|^{4}}\left\|d_{k-1}\right\|^{2}-2 \theta_{k} \frac{\left\|g_{k}\right\|^{2}}{g_{k-1}^{T} d_{k-1}} g_{k}^{T} d_{k-1}+2 \frac{\left\|g_{k}\right\|^{2}}{g_{k-1}^{T} d_{k-1}} g_{k}^{T} d_{k-1} \\
& =\left(\theta_{k}-1\right)^{2}\left\|g_{k}\right\|^{2}+\frac{\left\|g_{k}\right\|^{4}}{\left\|g_{k-1}\right\|^{4}}\left\|d_{k-1}\right\|^{2}-2 \frac{\left\|g_{k}\right\|^{2}}{\left(g_{k-1}^{T} d_{k-1}\right)^{2}}\left(g_{k}^{T} d_{k-1}\right)^{2}+2 \frac{\left\|g_{k}\right\|^{2}}{g_{k-1}^{T} d_{k-1}} g_{k}^{T} d_{k-1} \\
& \leq\left(\theta_{k}-1\right)^{2}\left\|g_{k}\right\|^{2}+\frac{\left\|g_{k}\right\|^{4}}{\left\|g_{k-1}\right\|^{4}}\left\|d_{k-1}\right\|^{2}+2 \frac{\left\|g_{k}\right\|^{2}}{g_{k-1}^{T} d_{k-1}} g_{k}^{T} d_{k-1} \cdot
\end{aligned}
$$


The above inequality along with (30) result

$$
\begin{aligned}
\left\|d_{k}\right\|^{2} & \leq\left(\theta_{k}-1\right)^{2}\left\|g_{k}\right\|^{2}+\frac{\left\|g_{k}\right\|^{4}}{\left\|g_{k-1}\right\|^{4}}\left\|d_{k-1}\right\|^{2}+2 c_{2} \frac{\left\|g_{k}\right\|^{2}}{g_{k-1}^{T} d_{k-1}} g_{k-1}^{T} d_{k-1} \\
& =\left(\theta_{k}-1\right)^{2}\left\|g_{k}\right\|^{2}+\frac{\left\|g_{k}\right\|^{4}}{\left\|g_{k-1}\right\|^{4}}\left\|d_{k-1}\right\|^{2}+2 c_{2}\left\|g_{k}\right\|^{2} .
\end{aligned}
$$

By dividing both sides of this inequality in $\left(g_{k}^{T} d_{k}\right)^{2}$ and using (22), we have

$$
\begin{aligned}
\frac{\left\|d_{k}\right\|^{2}}{\left(g_{k}^{T} d_{k}\right)^{2}} & \leq \frac{\left(\theta_{k}-1\right)^{2}\left\|g_{k}\right\|^{2}}{\left(g_{k}^{T} d_{k}\right)^{2}}+\frac{\left\|g_{k}\right\|^{4}\left\|d_{k-1}\right\|^{2}}{\left\|g_{k-1}\right\|^{4}\left(g_{k}^{T} d_{k}\right)^{2}}+\frac{2 c_{2}\left\|g_{k}\right\|^{2}}{\left(g_{k}^{T} d_{k}\right)^{2}} \\
& =\frac{\left(\theta_{k}-1\right)^{2}\left\|g_{k}\right\|^{2}}{\left\|g_{k}\right\|^{4}}+\frac{\left\|g_{k}\right\|^{4}\left\|d_{k-1}\right\|^{2}}{\left\|g_{k-1}\right\|^{4}\left\|g_{k}\right\|^{4}}+\frac{2 c_{2}\left\|g_{k}\right\|^{2}}{\left\|g_{k}\right\|^{4}} \\
& =\frac{\left(\theta_{k}-1\right)^{2}}{\left\|g_{k}\right\|^{2}}+\frac{\left\|d_{k-1}\right\|^{2}}{\left\|g_{k-1}\right\|^{4}}+\frac{2 c_{2}}{\left\|g_{k}\right\|^{2}} .
\end{aligned}
$$

By lemma $4,-2 \leq \theta_{k}-1 \leq 0$ and $0 \leq\left(\theta_{k}-1\right)^{2} \leq 4$. Hence

$$
\frac{\left\|d_{k}\right\|^{2}}{\left(g_{k}^{T} d_{k}\right)^{2}} \leq \frac{\left\|d_{k-1}\right\|^{2}}{\left(g_{k-1}^{T} d_{k-1}\right)^{2}}+\frac{\omega_{1}}{\left\|g_{k}\right\|^{2}}
$$

in which $\omega_{1}:=2\left(c_{2}+2\right)$. By applying (32) and (34), we can result

$$
\frac{\left\|d_{k}\right\|^{2}}{\left(g_{k}^{T} d_{k}\right)^{2}} \leq \frac{\left\|d_{k-1}\right\|^{2}}{\left(g_{k-1}^{T} d_{k-1}\right)^{2}}+\frac{\omega_{1}}{\left\|g_{k}\right\|^{2}} \leq \frac{\left\|d_{k-2}\right\|^{2}}{\left(g_{k-2}^{T} d_{k-2}\right)^{2}}+\frac{\omega_{1}}{\left\|g_{k-1}\right\|^{2}}+\frac{\omega_{1}}{\left\|g_{k}\right\|^{2}} \leq \cdots \leq \sum_{i=0}^{k} \frac{\omega_{1}}{\left\|g_{i}\right\|^{2}} \leq \frac{k \omega_{1}}{\varepsilon_{1}^{2}} \text {. }
$$

Therefore

Finally

$$
\frac{\left(g_{k}^{T} d_{k}\right)^{2}}{\left\|d_{k}\right\|^{2}} \geq \frac{\varepsilon_{1}^{2}}{\omega_{1}} \frac{1}{k} .
$$

which contradicts with Lemma 3.

$$
\sum_{k=0}^{\infty} \frac{\left(g_{k}^{T} d_{k}\right)^{2}}{\left\|d_{k}\right\|^{2}} \geq \frac{\varepsilon_{1}^{2}}{\omega_{1}} \sum_{k=0}^{\infty} \frac{1}{k}=+\infty
$$


Now, we investigate the convergence of MNTTCD algorithm in three cases. For $t_{k}=1$, this method reduces to NTTCD algorithm which its convergence established in Theorem 1 . Therefore, we prove other cases in the following theorem.

Theorem 2 Let $\left\{d_{k}\right\}_{k \geq 0}$ be a sufficient descent direction and $\left\{x_{k}\right\}_{k \geq 0}$ be the generated sequence by MNTTCD algorithm. Then

$$
\lim _{k \rightarrow \infty} \inf \left\|g_{k}\right\|=0 .
$$

Proof: We use contradiction to proof this theorem. Hence, there exists a constant $\varepsilon_{2}>0$ such that $\left\|g_{k}\right\|>\varepsilon_{2}$ for any $k$ and

Now (18), implies

$$
\frac{1}{\left\|g_{k}\right\|^{2}}>\frac{1}{\varepsilon_{2}^{2}}
$$

By substituting (4) and (17) in above equality, we get

$$
d_{k}=\left(t_{k} \theta_{k}-1\right) g_{k}+\beta_{k}^{C D} d_{k-1} .
$$

$$
\begin{gathered}
\left\|d_{k}\right\|^{2}=\left(t_{k} \theta_{k}-1\right)^{2}\left\|g_{k}\right\|^{2}+\left(\beta_{k}^{C D}\right)^{2}\left\|d_{k-1}\right\|^{2}+2\left(t_{k} \theta_{k}-1\right) \beta_{k}^{C D} g_{k}^{T} d_{k-1} \\
=\left(t_{k} \theta_{k}-1\right)^{2}\left\|g_{k}\right\|^{2}+\frac{\left\|g_{k}\right\|^{4}}{\left(g_{k-1}^{T} d_{k-1}\right)^{2}}\left\|d_{k-1}\right\|^{2}-2\left(t_{k} \theta_{k}-1\right) \frac{\left\|g_{k}\right\|^{2}}{g_{k-1}^{T} d_{k-1}} g_{k}^{T} d_{k-1} \\
=\left(t_{k} \theta_{k}-1\right)^{2}\left\|g_{k}\right\|^{2}+\frac{\left\|g_{k}\right\|^{4}}{\left(g_{k-1}^{T} d_{k-1}\right)^{2}}\left\|d_{k-1}\right\|^{2}-2 t_{k} \theta_{k} \frac{\left\|g_{k}\right\|^{2}}{g_{k-1}^{T} d_{k-1}} g_{k}^{T} d_{k-1} \\
+2 \frac{\left\|g_{k}\right\|^{2}}{g_{k-1}^{T} d_{k-1}} g_{k}^{T} d_{k-1}
\end{gathered}
$$

We consider two following cases:

Case (I) If $g_{k}^{T} d_{k-1}>0$, then $1<t_{k} \leq \eta_{1}$. Also, Lemma 3 implies

$$
\frac{1}{\left(g_{k}^{T} d_{k}\right)^{2}} \leq \frac{1}{\left\|g_{k}\right\|^{4}} \text {. }
$$

Now, Lemma 4 along with (17) give us $-1 \leq \theta_{k}<0$. From (37), we have

$$
\left\|d_{k}\right\|^{2} \leq\left(t_{k} \theta_{k}-1\right)^{2}\left\|g_{k}\right\|^{2}+\frac{\left\|g_{k}\right\|^{4}}{\left(g_{k-1}^{T} d_{k-1}\right)^{2}}\left\|d_{k-1}\right\|^{2} .
$$

We divide both sides of this inequality in $\left(g_{k}^{T} d_{k}\right)^{2}$ and use (38). Hence 


$$
\begin{aligned}
\frac{\left\|d_{k}\right\|^{2}}{\left(g_{k}^{T} d_{k}\right)^{2}} & \leq \frac{\left(t_{k} \theta_{k}-1\right)^{2}\left\|g_{k}\right\|^{2}}{\left(g_{k}^{T} d_{k}\right)^{2}}+\frac{\left\|g_{k}\right\|^{4}}{\left(g_{k-1}^{T} d_{k-1}\right)^{2}\left(g_{k}^{T} d_{k}\right)^{2}}\left\|d_{k-1}\right\|^{2} \\
& \leq \frac{\left(t_{k} \theta_{k}-1\right)^{2}\left\|g_{k}\right\|^{2}}{\left\|g_{k}\right\|^{4}}+\frac{\left\|g_{k}\right\|^{4}}{\left(g_{k-1}^{T} d_{k-1}\right)^{2}\left\|g_{k}\right\|^{4}}\left\|d_{k-1}\right\|^{2} \\
& =\frac{\left(t_{k} \theta_{k}-1\right)^{2}}{\left\|g_{k}\right\|^{2}}+\frac{\left\|d_{k-1}\right\|^{2}}{\left(g_{k-1}^{T} d_{k-1}\right)^{2}} .
\end{aligned}
$$

Since, $1<t_{k} \leq \eta_{1}$, we have

$$
\begin{aligned}
1<t_{k} \leq \eta_{1} & \Rightarrow \theta_{k} \eta_{1} \leq t_{k} \theta_{k}<\theta_{k}<0 \\
& \Rightarrow \theta_{k} \eta_{1}-1 \leq t_{k} \theta_{k}-1<-1 \\
& \Rightarrow-\eta_{1}-1 \leq t_{k} \theta_{k}-1<-1 \\
& \Rightarrow\left(t_{k} \theta_{k}-1\right)^{2} \leq\left(\eta_{1}+1\right)^{2}:=\omega_{2}
\end{aligned}
$$

This inequality and (39) result

$$
\frac{\left\|d_{k}\right\|^{2}}{\left(g_{k}^{T} d_{k}\right)^{2}} \leq \frac{\left\|d_{k-1}\right\|^{2}}{\left(g_{k-1}^{T} d_{k-1}\right)^{2}}+\frac{\omega_{2}}{\left\|g_{k}\right\|^{2}} .
$$

Case (II) If $g_{k}^{T} d_{k-1} \leq 0$, then $\eta_{2} \leq t_{k} \leq 0$. Also, Lemma 4 give us

Now, from (37), we have

$$
0 \leq \frac{g_{k}^{T} d_{k-1}}{g_{k-1}^{T} d_{k-1}} \leq 1 .
$$

$$
\left\|d_{k}\right\|^{2} \leq\left(t_{k} \theta_{k}-1\right)^{2}\left\|g_{k}\right\|^{2}+\frac{\left\|g_{k}\right\|^{4}}{\left(g_{k-1}^{T} d_{k-1}\right)^{2}}\left\|d_{k-1}\right\|^{2}-2 t_{k} \theta_{k}^{2}\left\|g_{k}\right\|^{2}+2\left\|g_{k}\right\|^{2} .
$$

By dividing both sides of this inequality in $\left(g_{k}^{T} d_{k}\right)^{2}$ and using (38)

$$
\begin{aligned}
\frac{\left\|d_{k}\right\|^{2}}{\left(g_{k}^{T} d_{k}\right)^{2}} & \leq \frac{\left(t_{k} \theta_{k}-1\right)^{2}\left\|g_{k}\right\|^{2}}{\left(g_{k}^{T} d_{k}\right)^{2}}+\frac{\left\|g_{k}\right\|^{4}}{\left(g_{k}^{T} d_{k}\right)^{2}\left(g_{k-1}^{T} d_{k-1}\right)^{2}}\left\|d_{k-1}\right\|^{2}+\frac{2\left(1-t_{k}\right)}{\left(g_{k}^{T} d_{k}\right)^{2}}\left\|g_{k}\right\|^{2} . \\
& \leq \frac{\left(t_{k} \theta_{k}-1\right)^{2}+2\left(1-t_{k}\right)}{\left\|g_{k}\right\|^{2}}+\frac{\left\|d_{k-1}\right\|^{2}}{\left(g_{k-1}^{T} d_{k-1}\right)^{2}} .
\end{aligned}
$$

Since $0 \leq \theta_{k} \leq 1$, we get

$$
\left(t_{k} \theta_{k}-1\right)^{2}+2\left(1-t_{k}\right)=t_{k}^{2} \theta_{k}^{2}-2 t_{k} \theta_{k}-2 t_{k}+3 \leq t_{k}^{2}-4 t_{k}+3=\left(t_{k}-1\right)^{2}-1,
$$
and 


$$
\begin{aligned}
\eta_{2} \leq t_{k} \leq 0 & \Rightarrow \eta_{2}-2 \leq t_{k}-2 \leq-2 \\
& \Rightarrow\left(t_{k}-2\right)^{2} \leq\left(\eta_{2}-2\right)^{2} \\
& \Rightarrow\left(t_{k}-2\right)^{2}-1 \leq\left(\eta_{2}-2\right)^{2}-1:=\omega_{3}
\end{aligned}
$$

By subsuiting this inequality to (40), we obtain

$$
\frac{\left\|d_{k}\right\|^{2}}{\left(g_{k}^{T} d_{k}\right)^{2}} \leq \frac{\left\|d_{k-1}\right\|^{2}}{\left(g_{k-1}^{T} d_{k-1}\right)^{2}}+\frac{\omega_{3}}{\left\|g_{k}\right\|^{2}} .
$$

Hence, in both cases similar to Theorem 1, we have

$$
\begin{gathered}
\frac{\left\|d_{k}\right\|^{2}}{\left(g_{k}^{T} d_{k}\right)^{2}} \leq \frac{\left\|d_{k-1}\right\|^{2}}{\left(g_{k-1}^{T} d_{k-1}\right)^{2}}+\frac{\omega_{j}}{\left\|g_{k}\right\|^{2}} \leq \frac{\left\|d_{k-2}\right\|^{2}}{\left(g_{k-2}^{T} d_{k-2}\right)^{2}}+\frac{\omega_{j}}{\left\|g_{k-1}\right\|^{2}}+\frac{\omega_{j}}{\left\|g_{k}\right\|^{2}} \\
\leq \cdots \leq \sum_{i=0}^{k} \frac{\omega_{j}}{\left\|g_{i}\right\|^{2}} \leq \frac{k \omega_{j}}{\varepsilon_{2}^{2}} \quad j=2,3 .
\end{gathered}
$$

Hence

Finally

$$
\frac{\left(g_{k}^{T} d_{k}\right)^{2}}{\left\|d_{k}\right\|^{2}} \geq \frac{\varepsilon_{2}^{2}}{\omega_{j}} \frac{1}{k} \quad j=2,3 .
$$

$$
\sum_{k=0}^{\infty} \frac{\left(g_{k}^{T} d_{k}\right)^{2}}{\left\|d_{k}\right\|^{2}} \geq \frac{\varepsilon_{2}^{2}}{\omega_{j}} \sum_{k=0}^{\infty} \frac{1}{k}=+\infty, \quad j=2,3 .
$$

Therefore, by this contradicts, the proof is complete.

\section{Numerical experiments}

In this section, we express numerical results on a set of some nonlinear unconstrained optimization test functions on the CUTEst collection [8] which are given in Table 1. The dimensions of test functions are from 2 to 12005 while the initial points are standard ones proposed in CUTEst. We apply the following algorithms to solve these test functions:

- FR: Fletcher-Reeves conjugate gradient method [16],

- HS: Hestenes-Stiefel conjugate gradient method [19],

- DY: Dai-Yuan conjugate gradient method [10],

- CD: Conjugate Descent conjugate gradient method [15],

- NTTCD: New three-term conjugate gradient method,

- MNTTCD: Modification of the new three-term conjugate gradient method.

All algorithms are implemented in Matlab 2011 programming environment on a $2.3 \mathrm{~Hz}$ Intel core i3 processor laptop and 4GB of RAM with the double precision data type in Linux operations system. The iterations stop whenever the inequality

$$
\left\|g_{k}\right\| \leq 10^{-6},
$$


be satisfied or the total number of iterates exceeds 10000 . Furthermore, we choose the parameters $\zeta_{1}=100, \zeta_{2}=50, \eta_{1}=15, \eta_{2}=-10, c_{1}=10^{-3}$ and $c_{2}=0.95$.

Here, we use the performance profiles of Dolan and More [12] to compare the performance of the algorithms on the test functions. We consider $P$ as designates the percentage of problems which are solved within a factor $\tau$ of the best solver. The horizontal axis of the figure gives the percentage of the test functions for which a method is the fastest (efficiency), while the vertical axis gives the percentage of the test functions that were successfully solved by each method (robustness).

Figures 1-3 show the performance of all algorithms to solve the unconstrained optimization problems. In these figures, $P(\tau)$ is designates the percentage of problems which are solved within a factor $\tau$ of the best solver. Figure 1 shows that the MNTTCD method wins about $32 \%$ of test problems with the smallest number of iterations. We conclude from Figure 2 that the NTTCD method is the most effective for most test functions in total number of function evaluations about $39 \%$. From figure 3, we can see that NTTCD method is better than other methods about $26 \%$ of the most wins in terms of CPU times.

\section{Conclusion}

In this work, we propose two three-term conjugate gradient directions based on CD conjugate gradient method. It is shown that the proposed directions always fulfil the sufficient descent property, independent of the line search. Under standard assumptions, we prove the convergence properties of the new schemes. The preliminary numerical experiment on a set of the test functions collection indicates that the new algorithms are effective.

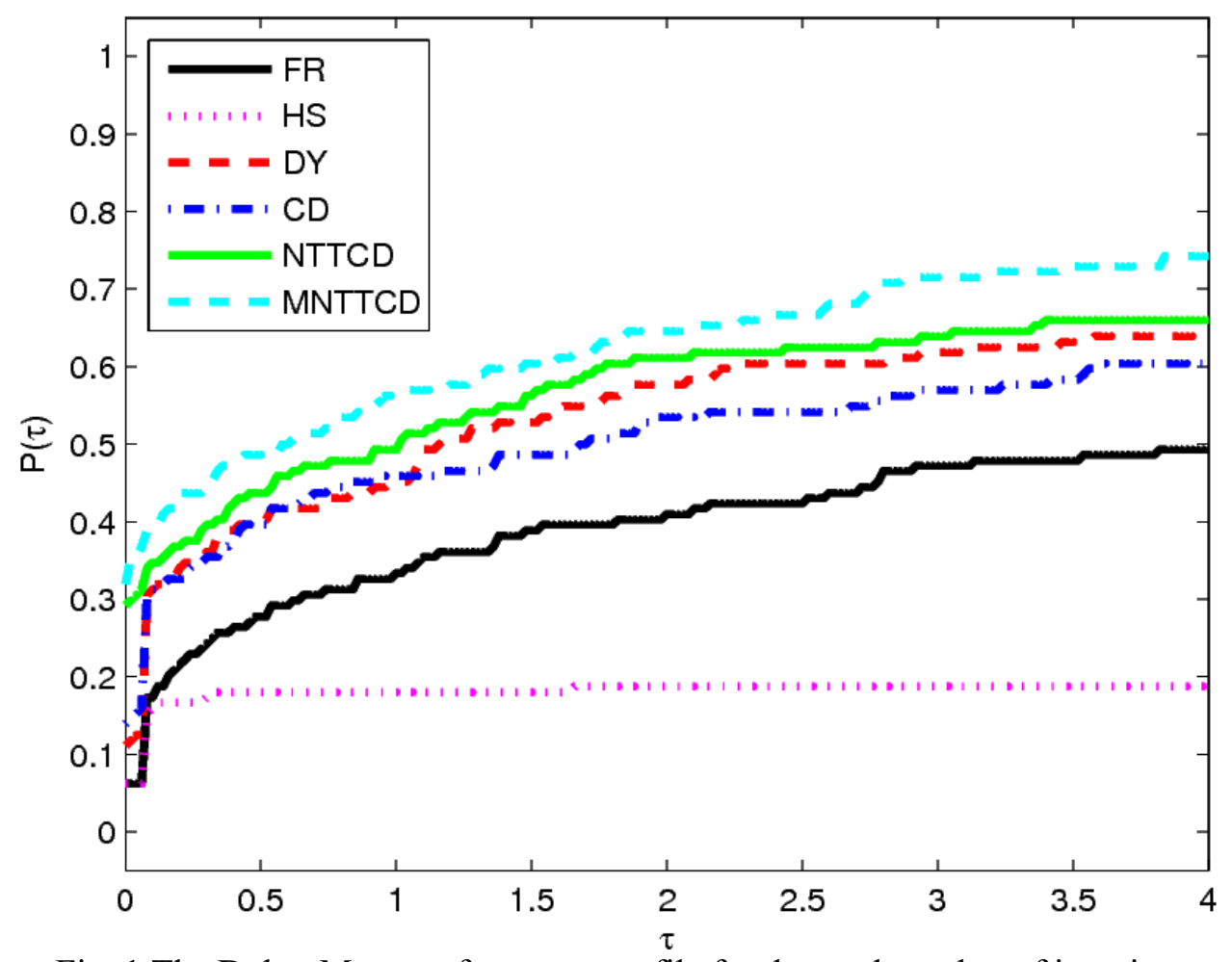

Fig. 1 The Dolan-More performance profile for the total number of iterations 


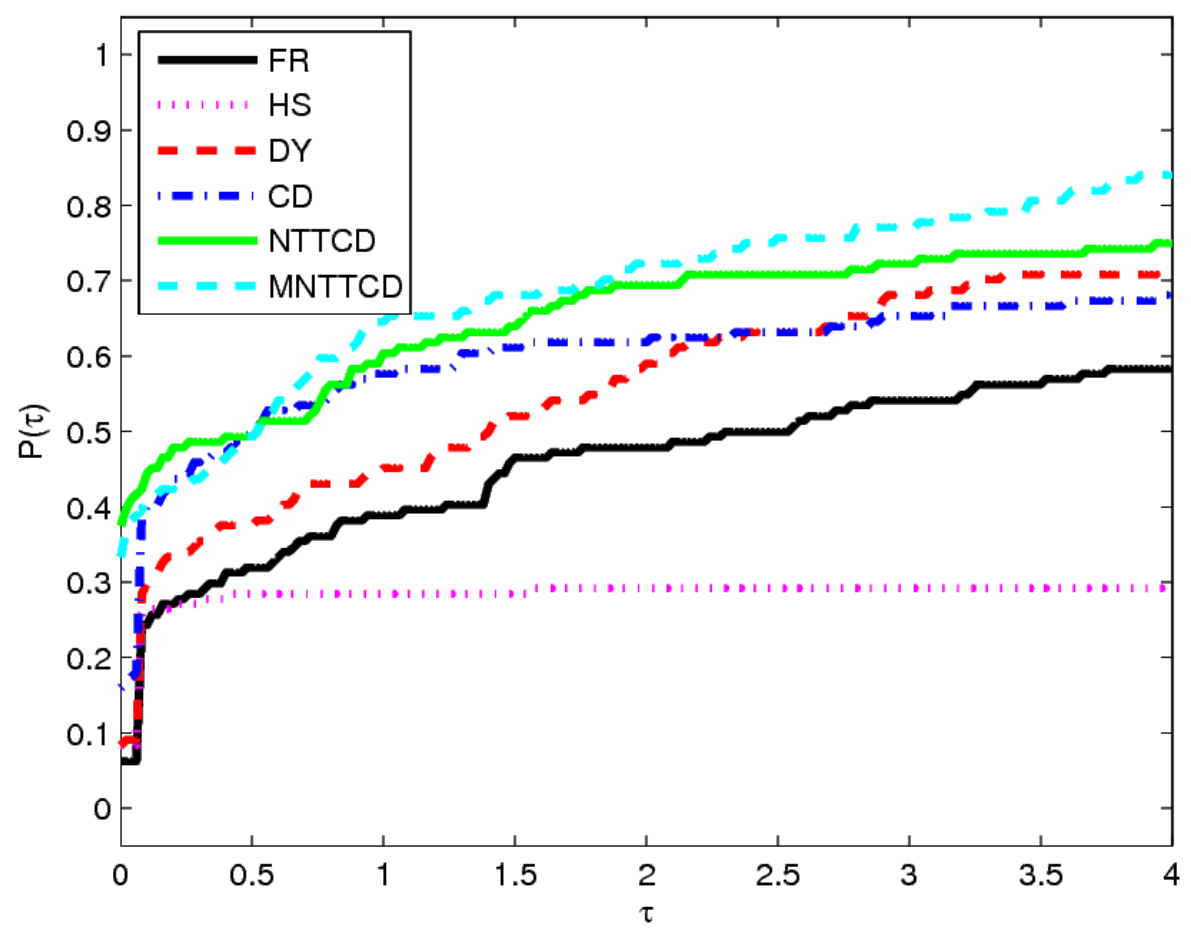

Fig. 2 The Dolan-More performance profile for the total number of functions evaluations

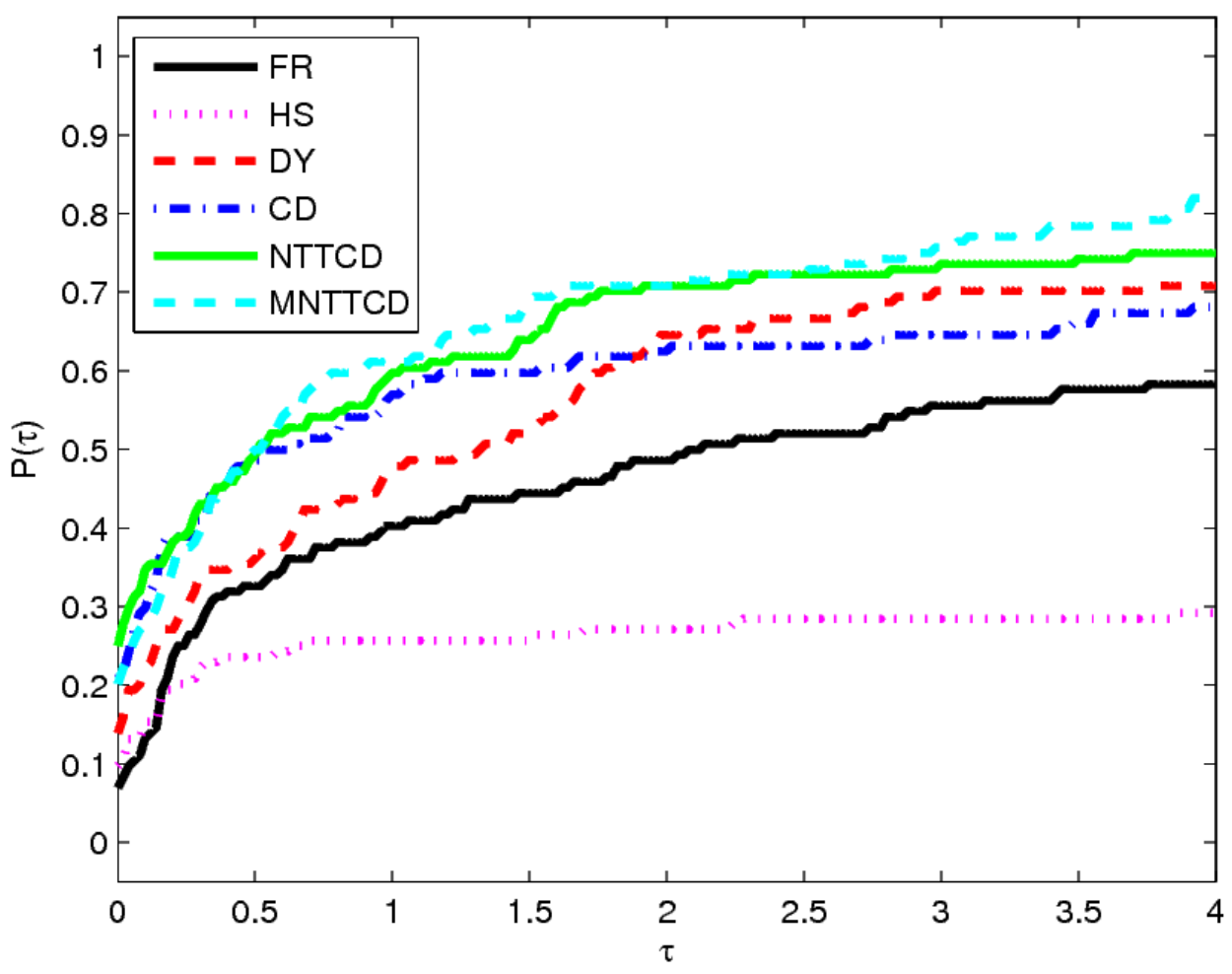

Fig. 3 The Dolan-More performance profile for the CPU times 
Table 1 Test functions taken from CUTEst collection

\begin{tabular}{|c|c|c|c|c|c|c|c|c|}
\hline No. & Test function & Dim & No. & Test function & Dim & No. & Test function & Dim \\
\hline 1 & 3PK & 30 & 49 & DQDRTIC & 10000 & 97 & NONDIA & 5000 \\
\hline 2 & AIRCRFTB & 8 & 50 & DQRTIC & 5000 & 98 & NONDQUAR & 5000 \\
\hline 3 & ALLINIT & 4 & 51 & EDENSCH & 100 & 99 & OSCIPANE & 5000 \\
\hline 4 & ALLINITU & 4 & 52 & EG2 & 1000 & 100 & OSCIPATH & 10 \\
\hline 5 & ARGLINA & 500 & 53 & EG3 & 10000 & 101 & OSLBQP & 8 \\
\hline 6 & ARGLINB & 200 & 54 & EIGENA & 2000 & 102 & PALMER1C & 8 \\
\hline 7 & ARWHEAD & 5000 & 55 & ENGVAL1 & 100 & 103 & PALMER1D & 7 \\
\hline 8 & BARD & 3 & 56 & ENGVAL2 & 3 & 104 & PALMER2C & 8 \\
\hline 9 & BDQRTIC & 100 & 57 & ERRINROS & 50 & 105 & PALMER3C & 8 \\
\hline 10 & BEALE & 2 & 58 & EXPFIT & 2 & 106 & PALMER4C & 8 \\
\hline 11 & BIGGS6 & 6 & 59 & EXTROSNB & 1000 & 107 & PALMER5C & 6 \\
\hline 12 & BIGGSB1 & 5000 & 60 & FLETCBV2 & 10000 & 108 & PALMER6C & 9 \\
\hline 13 & BOX2 & 3 & 61 & FLETCHCR & 500 & 109 & PALMER7C & 8 \\
\hline 14 & BOX3 & 3 & 62 & FMINSRF2 & 5625 & 110 & PALMER8A & 6 \\
\hline 15 & BRKMCC & 2 & 63 & FMINSURF & 5625 & 111 & PALMER8C & 8 \\
\hline 16 & BROWNDEN & 4 & 64 & FREUROTH & 2 & 112 & PENALTY1 & 100 \\
\hline 17 & BROYDN3D & 5000 & 65 & GENHUMPS & 5000 & 113 & PENALTY2 & 50 \\
\hline 18 & BROYDN7D & 500 & 66 & GENROSE & 500 & 114 & POWELLBC & 1000 \\
\hline 19 & BROYDNBD & 5000 & 67 & GROWTHLS & 3 & 115 & POWELLSG & 5000 \\
\hline 20 & BRYBND & 500 & 68 & GULF & 3 & 116 & QR3DLS & 610 \\
\hline 21 & CHAINWOO & 1000 & 69 & HAIRY & 2 & 117 & QUARTC & 25 \\
\hline 22 & CHNROSNB & 50 & 70 & HATFLDD & 3 & 118 & ROSENBR & 2 \\
\hline 23 & CLIFF & 2 & 71 & HATFLDF & 3 & 119 & S308 & 2 \\
\hline 24 & COSINE & 1000 & 72 & HATFLDFL & 3 & 120 & SCHMVETT & 100 \\
\hline 25 & CRAGGLVY & 1000 & 73 & HEART6LS & 6 & 121 & SENSORS & 100 \\
\hline 26 & CUBE & 2 & 74 & HEART8LS & 3 & 122 & SINEVAL & 2 \\
\hline 27 & CUBENE & 2 & 75 & HELIX & 3 & 123 & SINVALNE & 2 \\
\hline 28 & DALLASM & 196 & 76 & HILBERTA & 10 & 124 & SISSER & 2 \\
\hline 29 & DALLASS & 46 & 77 & HILBERTB & 1 & 125 & SNAIL & 2 \\
\hline 30 & DECONVU & 63 & 78 & HIMMELBA & 2 & 126 & SPARSINE & 1000 \\
\hline 31 & DENSCHNA & 2 & 79 & HIMMELBC & 2 & 127 & SPARSQUR & 10000 \\
\hline 32 & DENSCHNB & 2 & 80 & HIMMELBF & 4 & 128 & SPMSRTLS & 4999 \\
\hline 33 & DENSCHNC & 2 & 81 & HIMMELBG & 2 & 129 & SROSENBR & 1000 \\
\hline 34 & DENSCHNF & 2 & 82 & HIMMELBH & 2 & 130 & TAME & 2 \\
\hline 35 & DIXMAANA & 9000 & 83 & HUMPS & 2 & 131 & TESTQUAD & 100 \\
\hline 36 & DIXMAANB & 3000 & 84 & JENSMP & 2 & 132 & TOINTGOR & 50 \\
\hline 37 & DIXMAANC & 3000 & 85 & KOWOSB & 4 & 133 & TOINTGSS & 10000 \\
\hline 38 & DIXMAAND & 3000 & 86 & LIARWHD & 5000 & 134 & TOINTPSP & 50 \\
\hline 39 & DIXMAANE & 3000 & 87 & LOGHAIRY & 2 & 135 & TOINTQOR & 50 \\
\hline 40 & DIXMAANF & 3000 & 88 & MANCINO & 100 & 136 & TQUARTIC & 500 \\
\hline 41 & DIXMAANG & 3000 & 89 & MATRIX2 & 6 & 137 & TRIDIA & 5000 \\
\hline 42 & DIXMAANH & 3000 & 90 & METHANOL & 12005 & 138 & VAREIGVL & 500 \\
\hline 43 & DIXMAANI & 3000 & 91 & MODBEALE & 2 & 139 & VIBRBEAM & 8 \\
\hline 44 & DIXMAANJ & 3000 & 92 & MOREBV & 5000 & 140 & WATSON & 12 \\
\hline 45 & DIXMAANK & 3000 & 93 & MSQRTALS & 1024 & 141 & WEEDS & 3 \\
\hline 46 & DIXMAANL & 3000 & 94 & MSQRTBLS & 1024 & 142 & WOODS & 100 \\
\hline 47 & DIXON3DQ & 1000 & 95 & MINE5D & 10733 & 143 & YFITU & 3 \\
\hline 48 & DJTL & 2 & 96 & NONCVXU2 & 1000 & 144 & ZANGWIL2 & 2 \\
\hline
\end{tabular}




\section{References}

[1] M. Al-Baali, Descent property and global convergence of the Fletcher-Reeves method with inexact line search, IMA Journal of Numerical Analysis. 5, 121-124 (1985).

[2] M. Al-Baali, Y. Narushima, H. Yabe, A family of three-term conjugate gradient methods with sufficient descent property for unconstrained optimization, Computational Optimization and Applications. 60, 89-110 (2015).

[3] Z. Aminifard, S. Babaie-Kafaki, An optimal parameter choice for the Dai-Liao family of conjugate gradient methods by avoiding a direction of the maximum magnification by the search direction matrix, 4OR. 17, 317-330 (2019).

[4] N. Andrei, A new three-term conjugate gradient algorithm for unconstrained optimization, Numerical Algorithm. 68, 305--321 (2015).

[5] L. Arman, Y. Xu, M. Rostami, F. Rahpeymaii, Some three-term conjugate gradient methods for solving unconstrained optimization problems, Pacific Journal of Optimization. 16(3), 461-472 (2020).

[6] S. Babaie-Kafaki, A modified three-term conjugate gradient method with sufficient descent property, Applied Mathematics-A Journal of Chinese Universities. 30(3), 263-272 (2015).

[7] E. M. L. Beale, A derivative of conjugate gradients In: Lootsma, F.A (ed.) Numerical Methods for Nonlinear Optimization, Academic, London. 39-43 (1972).

[8] A. R. Conn, N. I. M. Gould, Ph. L. Toint, CUTE: constrained and unconstrained testing environment, ACM Transactions on Mathematical Software. 21, 123-160 (1995).

[9] Y. H. Dai, J. Y. Han, G. H. Liu, D. F. Sun, H. X. Yin, Y. Yuan, Convergence properties of nonlinear conjugate gradient methods, SIAM Journal on Optimization. 10, 345-358 (1999).

[10] Y. H. Dai, Y. Yuan, A nonlinear conjugate gradient method with a strong global convergence property, SIAM Journal on Optimization. 10, 177-182 (1999).

[11] S. Deng, Z. Wan, A three-term conjugate gradient algorithm for large-scale unconstrained optimization problems, Applied Numerical Mathematics. 92, 70-81 (2015).

[12] E. D. Dolan, J. J. More, Benchmarking optimization software with performance profiles, Mathematical Programming. 91, 201-213 (2002).

[13] X. L. Dong, D. R. Han, R. Ghanbari, X. L. Li, Z. F. Dai, Some new three-term Hestenes-Stiefel conjugate gradient methods with affine combination, Optimization. 66(5), 759-776 (2017).

[14] X. L. Dong, H. W. Liu, Y. B. He, New version of the three-term conjugate gradient method based on spectral scaling conjugacy condition that generates descent search direction, Applied Mathematics and Computation. 269, 606-617 (2015).

[15] R. Fletcher, Practical Methods of Optimization vol. 1: Unconstrained Optimization, John Wiley \& Sons, New York, 1987.

[16] R. Fletcher, C. Reeves, Function minimization by conjugate gradients, Computer Journal. 7(2), 149-154 (1964). 
[17] W. W. Hager, H. Zhang, A new conjugate gradient method with guaranteed descent and an efficient line search, SIAM Journal on Optimization. 16, 170-192 (2005).

[18] W. W. Hager, H. Zhang, A survey of nonlinear conjugate gradient methods, Pacific journal of Optimization. 2(1), 35-58 (2006).

[19] M. R. Hestenes, E. L. Stiefel, Methods of conjugate gradients for solving linear systems, Journal of research of the National Bureau of Standards. 49, 409-436 (1952).

[20] Y. Narushima, H. Yabe, J. A. Ford, A three-term conjugate gradient method with sufficient descent property for unconstrained optimization, SIAM Journal on Optimization. 21, 212-230 (2011).

[21] J. Nocedal, S. J. Wright, Numerical Optimization, Springer series in Operation Research, Springer, New York, 1999.

[22] E. Polak, G. Ribiere, Note sur la convergence de directions conjugees, Rev. Francaise Informat Recherche Opertionelle, 3e Annee. 16, 35-43 (1969).

[23] B. T. Polyak, The conjugate gradient method in extreme problems, USSR computational mathematics and mathematical physics. 9, 94-112 (1969).

[24] P. Wolfe, Convergence conditions for ascent methods, SIAM Review. 11, 226-235 (1968).

[25] P. Wolfe, Convergence conditions for ascent methods. II: some corrections, SIAM Review. 13(2), 185-188 (1971).

[26] G. Yuan, M. Zhang, A three-terms Polak-Ribiere-Polyak conjugate gradient algorithm for largescale nonlinear equations, Journal of Computational and Applied Mathematics. 286, 186-195 (2015).

[27] G. Zoutendijk, Nonlinear programming, computational methods. In: Abadie J (ed) Integer and nonlinear programming. Amsterdam, North-holland. 37-86 (1970). 